Itinéraires Itinéraires

Littérature, textes, cultures

2012-2 | 2012

Intime et politique

\title{
Le chronotope du salon dans quelques romans de mœurs parisiens (1860-1900)
}

Gabrielle Melison-Hirchwald

\section{(2) OpenEdition}

1 Journals

Édition électronique

URL : http://journals.openedition.org/itineraires/1101

DOI : 10.4000/itineraires. 1101

ISSN : 2427-920X

Éditeur

Pléiade

\section{Édition imprimée}

Date de publication : 1 novembre 2012

Pagination : 47-57

ISBN : 978-2-336-00027-5

ISSN : $2100-1340$

\section{Référence électronique}

Gabrielle Melison-Hirchwald, «Le chronotope du salon dans quelques romans de mœurs parisiens (1860-1900) », Itinéraires [En ligne], 2012-2 | 2012, mis en ligne le 01 novembre 2012, consulté le 10 décembre 2020. URL : http://journals.openedition.org/itineraires/1101 ; DOI : https://doi.org/10.4000/ itineraires. 1101

\section{(c) $(1)$}

Itinéraires est mis à disposition selon les termes de la licence Creative Commons Attribution - Pas d'Utilisation Commerciale - Pas de Modification 4.0 International. 


\title{
Le chronotope du salon dans quelques romans de mœurs parisiens (1860-1900)
}

\begin{abstract}
In the Parisian novel of manners of the second half of the nineteenth-century, as place of sociability, the drawing-room provides a fertile meeting ground where the private space of home is thrust onto the public stage. The salon chronotope furnishes the possibility to expose bourgeois speech and manners. Finally, as a mixed place, this room gives the opportunity to feminine characters to play an important role in politics in an underhand way.
\end{abstract}

Keywords : novel of manners, sociability, drawing-room, power, women Mots clés : roman de mœurs, sociabilité, salon, pouvoir, femmes

La première acception du substantif salon ${ }^{1}$ présentée par le Trésor de la langue française peut sembler commune mais elle place d'emblée cette pièce au cœur du foyer. Depuis son entrée dans la langue en 1650, l'emprunt à l'italien salone ( "grande salle ») connaît une fortune exceptionnelle dans la mesure où il désigne notamment par extension la demeure privée où la maîtresse de maison reçoit à jour fixe; et par métonymie ceux qui fréquentent ces réunions. Du règne de Louis XIII à la Belle Époque, de nombreux salons ont affiché une persistance durable, évoquant le mode de vie raffiné de la haute société, un lieu de communication spirituelle mais aussi l'endroit des divertissements superficiels. Ces représentations contrastées ont naturellement trouvé un écho dans les discours littéraires du $\mathrm{XIX}^{\mathrm{e}}$ siècle qui ont fabriqué l'image d'un salon cristallisé autour de figures mythifiées (Mme de Rambouillet, Mme Geoffrin, Mme Necker) et susceptible de se prêter à tous les investissements idéologiques². Dans la

1. «SALON [Dans un lieu privé] : pièce aménagée avec un soin particulier où l'on reçoit les visiteurs et où l'on se réunit en famille et entre amis ».

2. Antoine Lilti, Le Monde des salons. Sociabilité et mondanité à Paris au XVIII siècle, Paris, Fayard, 2005. 
fiction, de Stendhal à Proust, le salon joue le rôle d'un véritable chronotope au sens bakhtinien du terme : "lieu d'intersection des séries spatiales et temporelles du roman ${ }^{3} »$. Pour le romancier de mœurs, cet espace intermédiaire constitue une mine qu'il va exploiter sous diverses formes. En effet, pour mettre à jour ces « manières d'habiter ${ }^{4}$ » - à l'heure où la psychanalyse et la pipolisation de la vie publique sont des termes à inventer-, le salon offre cet endroit hybride entre l'intérieur et l'extérieur, l'officieux et l'officiel, le domestique et le politique, le secret et l'ostensible, idéal pour disséquer les habitudes sociales ${ }^{5}$.

Si l'approche sociocritique dessine une poétique du salon, il nous semble qu'une perspective lexicographique permettrait de mesurer plus précisément la place occupée par cette pièce particulière dans le roman de la seconde moitié du XIX ${ }^{e}$ siècle $^{6}$. Nous avons choisi un corpus (18601900) composé majoritairement de romans mondains et politiques se situant après Balzac, qui érige le salon en métonymie de l'élégance sociale, et avant Proust, qui va déterminer en profondeur la perception que l'on a des mondanités?

\section{Un espace de représentation}

Sas entre le dedans et le dehors, le salon est un lieu « entrouvert $^{8}$ » saturé de signes à décoder. Les substantifs « seuil », « porte », « frontières », « limites » préparent l'entrée et la sortie de la pièce tout comme les verbes de mouvement « traverser », « repasser », « introduire», " pénétrer», « entrer », « sortir ». Selon le contexte et la personne invitée, les serviteurs

3. Mikhail Bakhtine, Esthétique et théorie du roman, Paris, Gallimard, 1987, p. 387.

4. Michelle Perrot, Histoire de la vie privée, Paris, Seuil, 1999, t. IV, p. 281.

5. Afin de limiter notre propos, nous nous focaliserons principalement sur le terme salon en tant que pièce dans un lieu privé. Par moments, nous ferons toutefois référence à la dimension publique du substantif entendu comme salle de réception d'un établissement public et au Salon artistique au sens « d'exposition officielle des artistes vivants ».

6. Grâce à la base de données Frantext et au moteur de recherche Stella, nous avons pu mener un certain nombre d'analyses textuelles à partir du terme salon.

7. Corpus : Paul Bourget, Cruelle énigme, Paris, Lemerre, 1885; «Le Luxe des autres» dans Drames de famille, Paris, Plon, 1900; Jules Claretie, Candidat!, Paris, Dentu, 1887; Monsieur le Ministre, Paris, Dentu, 1881; Alphonse Daudet, Fromont jeune et Risler aîné; L'Immortel; Jack; Le Nabab; Numa Roumestan; Les Rois en exil, Paris, Gallimard, coll. « Bibliothèque de la Pléiade ", 1986-1994 et Soutien de famille, Paris, Librairie de France, 1929; Gustave Flaubert, L'Éducation sentimentale, Paris, Les Belles Lettres, 1942; Edmond et Jules de Goncourt, Charles Demailly (1864); Renée Mauperin (1876), Paris, Charpentier; Guy de Maupassant, Bel-Ami, Paris, Librairie de France, 1934; Notre Cour, Paris, Libraire de France, 1938; Émile Zola, La Curée; Son Excellence Eugène Rougon; Nana; Une page d'amour; Pot-Bouille; Le Ventre de Paris, Paris, Gallimard, coll. « Bibliothèque de la Pléiade », 1960-1964; L'Argent et L'CEuvre, Paris, Bernouard, 1928.

8. Guillaume Pinson, "Représentation et imaginaire des sociabilités au XIX siècle», Romantisme, $\mathrm{n}^{\circ} 143,2009$, p. 45. 
règlent le ballet des arrivées et des départs. Carrefour du domicile, le salon est le point de passage obligé où toutes les activités domestiques se concentrent : causeries, lectures, bals, repas, musique de chambre ${ }^{9}$. Il peut être tour à tour le lieu intime où la famille se réunit sous la lampe (la famille Joyeuse dans $\operatorname{Le~} N a b a b^{10}$ ), le lieu où l'on patiente (salon d'attente ${ }^{11}$ ), le lieu du paraître surtout.

Salle de réception, il est l'endroit où il faut être vu, même si cela impose des sacrifices quotidiens aux familles désargentées, comme les Josserand au début de Pot-Bouille ${ }^{12}$. «En France, l'honorabilité consiste [...] à avoir un salon splendide, quitte à coucher dans un galetas », note Texier, rédacteur en chef de L'Illustration et auteur d'une série de physiologies de Paris ${ }^{13}$. Le caractère ostentatoire ne concerne pas seulement le décor de la pièce mais un ensemble d'éléments qui font d'un salon un rendez-vous immanquable : la bonne adresse, la façade, les mets proposés, les sujets de conversation et bien sûr « la bonne compagnie, les gens du monde ${ }^{14} \gg$ qui y participent constituent des critères déterminants pour assurer la réussite de l'événement. Les obstacles peuvent donc être multiples. Parfois, la frontière ténue entre le domicile et le lieu de travail provoque une promiscuité embarrassante. Du salon de la baronne Hemerlingue, épouse d'un banquier, on perçoit « le tintement des pièces d'or, le fracas des piles d'écus écroulés sur les comptoirs » qui font « un accompagnement mercantile aux conversations susurrées par le catholicisme mondain ${ }^{15} »$.

À l'extérieur du foyer, le salon conserve ce statut intermédiaire. En témoigne le salon de restaurant offrant une intimité que n'ont pas les convives dans la salle commune ${ }^{16}$. C'est au café Riche que Renée cède pour la première fois à son beau-fils Maxime ${ }^{17}$. La même ambiance sensuelle envoûte les Forestier, Mme de Marelle et Duroy, « une vraie partie carrée » dans ces endroits « où les voisinages et les rencontres sont suspects ${ }^{18}$ ».

$\mathrm{Au}$ moyen de diverses strates, tout salon opère une sélection parmi ses habitués, les membres cultivant un entre soi où le pouvoir social est

9. Parfois, le salon permet de sortir sans être vu. La Curée, p. 480.

10. Le Nabab, chap. v.

11. Numa Roumestan, p. 46; Nana, p. 1267; Bel-Ami, p. 51-53.

12. Pot-Bouille, p. 26.

13. Voir la définition du terme «Salon » dans Pierre Larousse, Grand dictionnaire universel du XIX siècle, t. XIV, Paris, Administration du grand Dictionnaire, 1875, p. 133.

14. Par extension, il ne faut pas oublier que le salon désigne également ceux qui le fréquentent, Littré, 1862.

15. Le Nabab, p. 762.

16. Charles Demailly, p. 68; Bel-Ami, p. 79; Soutien de famille, p. 144 et p. 276; Jack, p. 389; Numa Roumestan, p. 190; Fromont jeune et Risler aîné, p. 941; Nana, p. 1300;

L'Euvre, p. 246.

17. La Curée, p. 456.

18. Bel-Ami, p. 254 et 256. 
aussi un pouvoir sur l'espace. Cependant, le triomphe de l'argent sous le Second Empire et la Troisième République contribue à modifier les pratiques sociales. Dans la fiction, se superposent deux représentations de l'ancien « salon de compagnie » : permanence du salon mondain et adaptation nécessaire aux nouveaux riches qui investissent les lieux ${ }^{19}$.

Le dialogue opposant Marthe à Charles dans Charles Demailly traduit bien la place ambiguë attribuée au salon dans la vie mondaine, entre héritage aristocratique et luxe tapageur du parvenu :

- Ah! Dis donc, reprit impitoyablement Marthe, tu n'as pas vu le salon que s'est fait arranger Voudenet... Il paraît qu'il gagne un argent! [...] Il a fait, cette année, une année d'au moins trente mille francs, c'est arrangé avec des ornements dorés...

- En pâte?

— En pâte ou en bois, je ne sais pas... et du velours cerise.

— Très beau! Quelque chose dans le genre d'un salon de dentiste américain acheté à la faillite d'un café des boulevards...

— Oh! D'abord, toi, tu n'aimes que tes vieilleries et tes bibelots... tout ce qui n'est pas vieux... et puis tu ne veux pas que les femmes aient du goût... je sais cela ${ }^{20}$.

Mise à part la pointe misogyne finale caractéristique de l'écriture goncourtienne, ce conflit de valeurs traverse l'ensemble du corpus. Dans le « petit salon paisible », à la fois "sévère et intime », de Mme Castel et de sa fille Mme Liauran, trône une pendule offerte par Napoléon I ${ }^{\mathrm{er}}$. Les deux femmes figées dans le culte des souvenirs tentent toutefois de plaire au jeune Hubert Liauran en lui aménageant un espace indépendant, moderne et confortable. Mais c'est compter sans le salon de Mme de Sauve qui attire irrésistiblement le jeune homme épris de la maîtresse de maison. Cet antagonisme se répète à l'envi lors de scènes de genre mises en miroir : la solennité du salon de l'hôtel Béraud et le salon blanc et or de l'hôtel Saccard $^{21}$; le salon des Le Quesnoy et le salon des Roumestan ${ }^{22}$; le salon des Arnoux et de Dambreuse ${ }^{23}$; le salon « sépulcral ${ }^{24}$ » de Sabine Muffat (chap. III) et le salon de Nana (chap. IV); le salon de Saccard et de celui des Hamelin ${ }^{25}$.

Ces « manières d'habiter » montrent à quel point le salon dépeint indirectement le caractère et le statut social des personnages. Les viveurs

19. Arno Mayer, La Persistance de l'Ancien Régime, l'Europe de 1848 à la Grande Guerre, Paris, Flammarion, 1983.

20. Charles Demailly, p. 243.

21. La Curée, p. 503 et p. 522.

22. Numa Roumestan, p. 22 et 194.

23. L'Éducation sentimentale, p. 102.

24. Le rapprochement entre l'église et le salon n'est pas rare, en particulier chez Zola. Pot-Bouille, p. $364 ;$ La Curée, p. 412.

25. L'Argent, p. 51 et p. 61. 
s'opposant apparemment aux ascètes, la grande bourgeoisie et la noblesse aux commerçants enrichis. Cette dichotomie existait déjà du temps de Balzac, dès l'incipit de La Cousine Bette, par exemple, qui met aux prises Crevel avec la baronne Hulot. Mais le siècle avançant, le salon devient plus ouvert. Il demeure pourtant un espace clos duquel les intrus se trouvent exclus. Ce caractère se vérifie dans tous les milieux, qu'il s'agisse du peuple ${ }^{26}$, du demi-monde et du monde (Nana, Charles Demailly). Cultiver l'entre soi suppose quelques règles auxquelles on ne saurait déroger : accès restreint et sélection en fonction de la richesse, de la naissance, de l'entregent et des talents particuliers des membres. C'est un monde qui a «ses exclusions, ses mœurs, sa hiérarchie ${ }^{27} »$.

On remarque en effet l'ambiguïté du terme "salon» à travers les synonymes qui lui sont corrélés : « cabinet, cercle, pièce, musée, fumoir, foire, exposition, entourage, club, boudoir, bal et ruelle ${ }^{28} \gg$. Si les substantifs « ruelle » et « musée » peuvent sembler anachroniques dans le roman du XIX ${ }^{\mathrm{e}}$ siècle ${ }^{29}$, le terme « salon » reste sans doute le plus générique pour appréhender une salle de réception dans le corpus. Il n'est donc pas étonnant que par rapport aux autres pièces du foyer, le salon soit l'endroit le plus cité avec la chambre, loin devant le cabinet, la cuisine, la salle à manger, l'antichambre, le boudoir et la salle de bains. Notons la polysémie des trois premiers lieux se situant soit dans l'espace du dehors (Chambre des députés, Salon des Refusés, cabinet de ministre), soit dans l'espace du dedans (chambre à coucher, petit salon, cabinet particulier, cabinet de toilette, etc.). Cependant, il s'agit pour toutes ces affectations diverses de lieux fermés à accès restreint dont l'ouverture se limite à des habitués, des initiés, voire des intimes.

En contexte, le salon est donc rarement un lieu insignifiant. À part le salon d'Ida de Barancy «banal comme un salon de dentiste avec un ciel bleu $^{30} »$, la pièce de réception revêt un caractère particulier dans le roman de mœurs. Le salon officialise des scènes familiales ou intimes que le narrateur se plaît à évoquer. Là encore, la dimension morale réapparaît avec d'autant plus de force. Le salon est tour à tour le home dans lequel les familles trouvent refuge et le lieu du vice qui place le lecteur en position de voyeur. Le salon peut représenter en effet le havre de paix que connaissent les personnages loin de l'agitation parisienne. "Tranquille refuge épargné », " calme asile », telles sont les périphrases utilisées pour désigner ces lieux où les rumeurs de la capitale ne parviennent que de façon étouffée ${ }^{31}$. Parfois,

26. Soutien de famille, p. 277.

27. « Le Luxe des autres", p. 84.

28. Dictionnaire des synonymes CRISCO, [En ligne], http://www.cnrtl.fr/synonymie/.

29. Notons toutefois que M. Walter constitue son musée privé dans ce qu'il nomme son « Salon carré ». Bel-Ami, p. 134.

30. Jack, p. 73.

31. Le Nabab, p. 557 ; Cruelle Énigme, p. 6. 
il s'agit de l'évocation nostalgique provinciale d'un passé reconstruit loin de « la cohue de Paris ${ }^{32}$ ». En contrepoint, le petit salon bouton d'or abrite les amours incestueuses de Renée et de Maxime, mettant le lecteur dans le secret de l'alcôve; le salon japonais de Mme Marsy réunit dans un tête-àtête brûlant le duc de Rosas et la courtisane Marianne Kayser ${ }^{33}$.

\section{Une scène de genre ritualisée}

Le salon peut constituer un espace très différent d'une scène de genre à une autre. C'est pourquoi d'aucuns le définissent parfois par la négative : " Pièce qui ne sert ni de cabinet, ni de chambre à coucher, où l'on peut se réunir ${ }^{34} »$. Réside dans ce dernier verbe l'intérêt de l'endroit. Propice à rassembler les personnages, le salon offre l'occasion de voir s'y dérouler une scène décisive de l'action tout en s'inscrivant de manière plus globale dans un processus de ritualisation. Les termes les plus souvent associés au substantif «salon » au sein d'une même phrase renvoient d'abord à l'espace (adjectifs petit [174 occurrences] et grand [145]), la temporalité apparaissant en second plan (jour [46]; soir [35]; moment [31]; heures $[28])^{35}$. Le salon constitue non seulement un espace réel et fantasmatique mais obéit également à un temps particulier ritualisé et aux fonctions diversement marquées selon la saison, le moment de la semaine ou l'heure de la journée. Si l'espace est abondamment décrit, le caractère temporel se manifeste moins par l'emploi d'expressions comme « tenir salon », " faire salon », « fréquenter un salon » que par l'aspect itératif indiquant le jour de réception.

En effet, le salon «suppose un jour ${ }^{36} »$ : Sabine Muffat et Mme le Prieux reçoivent le mardi ${ }^{37}$, Renée les jeudi et lundi ${ }^{38}$, Rougon, les dimanche et jeudi. On dîne chez Sandoz chaque jeudi ${ }^{39}$. L'échéance est également annuelle. Au mois de janvier, Mme Deberle donne un bal dans son grand salon noir et or (Une Page d'amour). Le Salon artistique obéit à la même exigence : l'ensemble des artistes a cette date butoir en tête dans $L^{\prime} E^{\prime}$ uvre ${ }^{40}$. Elle peut être enfin l'occasion de fêter un événement particulier. Les somptueux salons de l'hôtel de Rosen ne furent ouverts qu'à l'occasion

32. Monsieur le Ministre, p. 42-43.

33. Ibid., p. 122.

34. François Raymond, Dictionnaire général de la langue française et vocabulaire universel des sciences, des arts et des métiers, Paris, A. André, 1832.

35. L'étude de voisinage a été opérée sur 21 des 24 romans du corpus car les deux romans de Claretie et « Le Luxe des autres » de Bourget ne figurent pas encore dans la base Frantext. 36. «Le Luxe des autres », p. 103.

37. Nana, p. 1144; «Le Luxe des autres », p. 164 sq. Voir chap. v : «Le jour de Madame le Prieux ».

38. La Curée, p. 444 et 493.

39. Charles Demailly, p. 178; Nana, p. 1364.

40. L'Euvre, p. 32, 87, 113, 117, 119, 121, 125, 255, 278, 281, 285, 291. 
du mariage d'Herbert ${ }^{41}$. Rue de Villiers, Nana réserve le grand salon « du Louis XVI trop riche " aux " soirs de gala ${ }^{42}$ ». Dans ce cas, la scène de genre se déploie sur plusieurs pages, certains chapitres étant dévolus à des scènes prandiales et à des conversations mondaines.

Dans cette pièce hautement théâtrale qu'est le salon ${ }^{43}$ alternent les tonalités qui vont du pur comique aux situations les plus poignantes. Par exemple, le salon désert des rois d'Illyrie semble " prêt pour quelque scène tragique » alors que la reine Frédérique ruinée cherche un prêteur sur gages auprès de Mérault. Une fois les convives réunis, lorsque la scène est développée, cela donne lieu à des conversations dont la teneur est parfois simplement résumée, parfois retranscrite par bribes en fonction des personnages présents, de leurs déplacements selon qu'ils prennent part à la conversation ou qu'ils observent celle des autres.

De quoi parle-t-on? La première du vaudeville, les confidences sur les domestiques, les travaux d'Haussmann à Paris, le prochain Salon officiel, la candidature d'un écrivain à l'Académie... L'actualité politique est abordée de façon biaisée. Dans Nana on évoque Bismarck à la fois dans le salon de Sabine Muffat et dans celui de Nana ${ }^{44}$. Entre propos lestes, situation économique et politique, vie mondaine, le narrateur évolue de groupe en groupe pour saisir au vol le contenu des conversations. L'instance narrative est en général secondée par un personnage outsider qui, sous les apparences, dévoile les mœurs véritables des protagonistes. C'est par le regard de Mouret ou de Liauran que l'atmosphère du salon nous est relayée. L'hypocrisie sociale à travers les caractères factice, illusoire et éphémère des rapports humains transpire dans chacun des romans du corpus à l'occasion de cette scène de genre.

À partir d'une volonté a priori réaliste, le roman de mœurs aboutit à une multitude de configurations possibles reflétées par les points de vue antagonistes des personnages. Le dernier dîner de Sandoz met en exergue les tensions, rivalités et trahisons qui agitent les artistes ${ }^{45}$. Certains se plient à ces conventions comme Couturat qui devient le "Couturat des salons", « un Couturat que ses amis ne connaissaient pas ${ }^{46}$ » : l'abbé Blampoix, n'ayant ni cure ni paroisse, se spécialise dans la confession des salons ${ }^{47}$. D'autres ne les supportent pas, tels Mme Grandjean, la provinciale Adrienne Vaudrey, les jeunes filles possédant leur franc-parler ou le naïf Paul de Géry.

41. Les Rois en exil, p. 904. Par économie, les Beauvilliers ferment les salons quand elles n'attendent personne. L'Argent, p. 70.

42. Nana, p. 1348.

43. La description du salon de Mme Marsy en est un exemple parmi d'autres. Monsieur le Ministre, p. 89.

44. Nana, p. 1147 et 1178.

45. L'Euvre, p. 366.

46. Charles Demailly, p. 17.

47. Renée Mauperin, p. 67. 
Les salons apparaissent comme une formidable caisse de résonance, parfois amplifiée par le relais de la presse. Médiatisés, ils sont « comme ces machines qui prennent la matière à l'état brut et la rendent centuplée de valeur ${ }^{48}$ ». Dans l'économie du roman, le salon est un espace modulable qui s'adapte à l'usage qu'on en fait. Il est d'abord à l'image de ses propriétaires : mobilier dépareillé et luxe extravagant chez Clorinde Balbi, « meubles méthodiquement rangés », " austérité presque religieuse d'un salon de couvent » chez les Rougon ${ }^{49}$. Selon le statut du convive, la pièce change de fonction. Amant de Michèle de Burne, André Mariolle passe ainsi du salon mondain ouvert à tous les prétendants de la maîtresse de maison au « salon des intimes ${ }^{50} »$.

De façon globale, les scènes et les conversations se font écho. Parfois, seuls les convives changent ${ }^{51}$. Les scènes de genre se dupliquent dans un mimétisme factice. Chez Blanche, Renée repère les lieux pour les décrire à ses intimes. Elle y côtoie « les mêmes financiers, les mêmes hommes politiques, les mêmes jeunes viveurs qui venaient à ses jeudis. Elle se croyait dans son salon ${ }^{52} \gg$. Cette reproduction à l'identique transgresse les barrières sociales : imitation entre la salle à manger de Rosanette et le salon de Mme Arnoux $^{53}$. Réplique punitive enfin pour Duveyrier : alors qu'il appréciait l'atmosphère beaucoup plus libre du salon de sa maîtresse, il retrouve en pire ce qu'il connaissait déjà : « un coin de bourgeoisie féroce, où il retrouvait tous les ennuis de son ménage, dans de l'ordure et du vacarme ${ }^{54} \gg$. Au fur et à mesure de l'intrigue, le vernis des apparences s'écaille. Le masque mondain tombe souvent de manière brutale. Si le salon de Sabine Muffat demeure tel que sa belle-mère le connaissait de son vivant, il ne saurait cacher longtemps l'infidélité de l'épouse.

De manière réflexive, évoquer le salon révèle aussi une prise de conscience de la part des protagonistes. Dans sa retraite à Fontainebleau, André Mariolle se souvient avec nostalgie des causeries dans le salon de Michèle de Burne ${ }^{55}$. À la fin de Pot-Bouille, « Octave eut une singulière sensation de recommencement ${ }^{56} \gg$. Risler retrouve à la fin du roman le salon de chez Véfour où il s'est marié ${ }^{57}$. La scène de genre met progressivement à nu les actes véritables des personnages. Cependant, l'instance narrative ne cherche pas à fouiller leur intimité comme dans le roman psychologique. Il

48. L'Éducation sentimentale, p. 102.

49. Son Excellence Eugène Rougon, p. 130.

50. Notre Cour, p. 407.

51. Nana, p. 1178.

52. La Curée, p. 444-445.

53. L'Éducation sentimentale, p. 185.

54. Pot-Bouille, p. 309-310.

55. Notre Coeur, p. 465 et p. 496.

56. Pot-Bouille, p. 381.

57. Fromont jeune et Risler aîné, p. 1170. 
s'agit de montrer par la réunion des protagonistes le caractère artificiel d'un monde qui ne croit plus en ses valeurs.

\section{Le terrain d'élection des femmes}

Les lieux élus « chambre » et « salon » conduisent à une mise en évidence de la vie privée dans la fiction. Même dans les romans à prétexte politique, très peu de scènes se situent dans les espaces attendus de la vie publique (Chambre des députés, ministère) ${ }^{58}$. Non qu'elle n'intéresse pas les protagonistes car celle-ci se trouve imbriquée dans le domestique : «L'Opéra, mais cela fait partie de la politique! » soutient le banquier Molina ${ }^{59}$. Dans 1'exercice du pouvoir, les ambitieux Rougon, Jansoulet, Vaudrey, Duroy ont en commun d'être surtout décrits dans leur vie personnelle.

« La clef de la situation donne très souvent sur les coulisses ${ }^{60}$ ». Lorsqu'un lieu public est toutefois exposé, il l'est souvent de l'intérieur ainsi qu'en témoigne l'attaque antiparlementaire menée par Izoard, sténographe à 1 'Assemblée ${ }^{61}$. Le salon fait évidemment partie de cette stratégie de conquête de pouvoir. Ceux qui l'ont perdu se réfugient dans cet espace privé : dans Les Rois en exil, se retrouvent, dans « l'étroit salon de la banlieue de Paris », des « princes déchus » qui n’ont plus de territoire pour régner ${ }^{62}$. Le duc de Mora reçoit chez lui dans son salon et tient ses audiences dans sa chambre à coucher ${ }^{63}$. Le salon lui-même se publicise : « Dès le lendemain le conseil se réunit à l'hôtel d'Orviedo, dans l'ancien salon de Saccard, transformé en salle des séances ${ }^{64}$. »

Dans cette survalorisation de la vie privée, il n'est pas étonnant de constater que le terme « femme » arrive en tête des substantifs figurant dans les romans du corpus. Au XIX ${ }^{\mathrm{e}}$ siècle, le salon constitue le seul espace mixte au sein duquel les personnages féminins peuvent exercer quelque influence dans la vie publique. Sous le Second Empire et la Troisième République, la plupart des salons étaient tenus par des femmes. Les salons de la princesse Mathilde, de Mme de Ratazzi puis ceux de Mmes d'Aubernon ou de Loynes permettent d'imbriquer mondanités et sphère privée. Cette prépondérance féminine se retrouve dans la fiction.

58. Lorsque ces lieux sont mentionnés, c'est souvent pour y évoquer une disgrâce : démission d'Eugène Rougon à la Chambre des députés (Son Excellence Eugène Rougon, chap. I); séance d'invalidation du député Jansoulet (Le Nabab, chap. XxI).

59. Monsieur le Ministre, p. 8-9.

60. Ibid.

61. Soutien de famille, chap. IX.

62. Les Rois en exil, p. 935.

63. Le Nabab, p. 484.

64. L'Argent, p. 139. 
Le salon peut par exemple constituer le théâtre où les mariages se font et se défont. Dans les milieux de la noblesse et de la bourgeoisie, le marriage market coupe court à la romantic comedy. "C'était la rage de ce salon, un furieux appétit de gendres, qui dévorait ces bourgeoises ${ }^{65} \gg$. Cependant, l'influence féminine dépasse ces tractations matrimoniales dans la mesure où grâce à son entregent, la femme tisse le réseau relationnel nécessaire pour affermir un statut social, construire une carrière, asseoir une réputation. Courtisanes et bourgeoises tirent parti de leurs qualités respectives. L'affrontement politique passe alors par le salon où les femmes servent de médiatrices. Ainsi, la lutte entre Rougon et Marsy s'exprime par une joute verbale « que les dames colportaient dans les salons ${ }^{66}$ ». Le décolleté de Renée sert les intérêts politiques de son époux Saccard ${ }^{67}$. C'est pourquoi, chaque femme tenant salon s'efforce d'inviter des célébrités qui pourront aider ceux qu'elles défendent : le duc de Rosas et le ministre de l'Intérieur sont courtisés par Sabine Marsy; Michèle de Burne cherche à attirer le sculpteur Prédolé. Conscients de ce rôle en sous-main, les personnages masculins s'appuient sur les relations de leurs épouses et de leurs maîtresses. Bel-Ami met en œuvre cette stratégie et le salon de son épouse va l'aider pour un temps dans sa fulgurante ascension sociale : "A tout moment, il trouvait dans son salon, en rentrant chez lui, un sénateur, un député, un magistrat, un général, qui traitaient Madeleine en vieille amie, avec une familiarité sérieuse ${ }^{68}$. " Les éternels prétendants à la Coupole hantent les salons académiques qui « préparent les élections, pointent les voix, et dont la fréquentation vaut mieux pour un candidat que son pesant de génie ${ }^{69} »$. La charge est plus virulente dans L'Immortel où la nullité des impétrants se voit compensée par l'habileté féminine : "Le salon de la duchesse [Padovani] leur a rudement servi à tous ", souligne l'ambitieux Paul Astier ${ }^{70}$.

Toutefois, les hommes sont aussi victimes des ruses féminines. « Gare alle donne » avait prévenu Ramel à l'attention de Vaudrey. «Les femmes, qui se plaignent de n'avoir pas de droits politiques, les ont tous, en réalité, puisqu'elles ont le pouvoir de diriger les ministères et de faire sauter les ministres, comme la Du Barry ses oranges !... ${ }^{71} \gg$ Et Claretie de reprendre l'antienne dans Candidat! en nommant celle qui agit dans l'ombre : «C'est Mme Herblay! Elle a fait et défait Javouillet, elle a fait Charvet, elle fera son successeur ${ }^{72} \gg$.

65. Pot-Bouille, p. 50.

66. Son Excellence Eugène Rougon, p. 132.

67. La Curée, p. 475.

68. Ibid., p. 234.

69. Les Rois en exil, p. 1011.

70. L'Immortel, p. 703.

71. Monsieur le Ministre, p. 179.

72. Candidat!, p. 35. 
Aboutissant à une publicisation de l'intime et à une privatisation du public, le salon se trouve d'autant plus dramatisé qu'il correspond fréquemment à une scène décisive du point de vue de la narration. Dans les romans à prétexte politique, il se substitue aux lieux publics traditionnels en tant qu'espace de débat. Lieu des relations mondaines, intimes ou familiales, le salon offre un espace intermédiaire où le rituel se maintient même au sein d'une société en perpétuelle évolution. Chaque salon a sa couleur au sens littéral du terme, qui lui confère une assise politique et morale. Les convives changent de nom au gré des chutes de gouvernement et des élections académiques. Mais les codes se perpétuent dans l'apparent paradoxe de la répétition et de la mise en exergue de l'événement. Le romancier de mœurs accorde enfin une tribune aux personnages féminins ordinairement exclus de la vie publique en leur permettant d'agir en sous-main dans cette antichambre du pouvoir formée par le salon. Romancier des apparences, le romancier de mœurs étudie dans le laboratoire qu'est le salon les pratiques et conduites sociales, mais avec un art du compromis qui lui fait éviter toute forme d'engagement et d'analyse des comportements. Entre intime et politique en somme pour ne pas prendre parti.

Gabrielle Melison-Hirchwald Université de Lorraine, ATILF-CNRS (UMR 7118) 